\title{
Über die Problematik der klinischen Entscheidungsfindung aufgrund von Fallbeschreibungen - ethische Implikationen am Beispiel eines Falls von Carmi Syndrom
}

\author{
Oliver J. Muensterer $(\mathbb{D}) \cdot$ Norbert W. Paul
}

Eingegangen: 27. April 2020 / Angenommen: 15. Mai 2020 / Online publiziert: 30. Juni 2020

(C) Der/die Autor(en) 2020

Zusammenfassung Bei extrem seltenen Erkrankungen bilden Fallbeschreibungen oft die einzige Datengrundlage für klinische Entscheidungen. Das Carmi Syndrom ist eine seltene Kombination von Epidermolysis bullosa und Pylorusatresie. Während der Betreuung einer betroffenen Patientin fielen unterschiedliche Wahrnehmungen über die publizierten Mortalitätsraten auf. Daraufhin wurde die Hypothese untersucht, ob sich die kumulativen Mortalitätsraten von Einzelfallbeschreibungen und Mehrfachfallbeschreibungen unterscheiden, um so eine mögliche Verzerrung der Prognose in ihren Auswirkungen auf klinische und ethische Einschätzungen des Falls zu überprüfen.

Ein Mädchen wurde in der Schwangerschaftswoche 33 mit Carmi Syndrom geboren. Zusammen mit dem klinischen Ethikkomitee wurden Behandlungsoptionen diskutiert, einschließlich einer palliativen Behandlung oder einer operativen Gastrojejunostomie. Da etwa ein Drittel der in Fallbeschreibungen publizierten Kinder nach einer Operation überlebten, entschieden wir uns auch vor dem Hintergrund unsicherer Prognosen für das chirurgische Vorgehen. Die Patientin starb 4 Wochen später nach multiplen Komplikationen.

Die Datenbank PubMed wurde nach Publikationen über Carmi Syndrom durchsucht. Das Outcome von Einzelfallbeschreibungen wurde mit dem von Mehrfachfallbeschreibungen verglichen.

\footnotetext{
Univ.-Prof. Dr. med. O. J. Muensterer ( $\square)$

Klinik und Poliklinik für Kinderchirurgie, Universitätsmedizin Mainz der Johannes Gutenberg Universität, Langenbeckstraße 1, 55131 Mainz, Deutschland

E-Mail: oliver.muensterer@unimedizin-mainz.de
}

Univ.-Prof. Dr. med. O. J. Muensterer · Univ.-Prof. Dr. rer. med. N. W. Paul, M.A.

Institut für Geschichte, Theorie und Ethik der Medizin, Universitätsmedizin Mainz, Mainz,

Deutschland

Univ.-Prof. Dr. rer. med. N. W. Paul, M.A.

Klinisches Ethikkomitee, Universitätsmedizin Mainz, Mainz, Deutschland 
Insgesamt wurden 102 Fälle von Carmi Syndrom identifiziert. Die Mortalität bei Einzelfallbeschreibungen belief sich auf 17 von 27 Fällen (63\%), während 62 von 74 Patienten von Mehrfachfallbeschreibungen starben $(84 \%, p=0,036)$.

Beim Carmi Syndrom unterscheidet sich die publizierte Mortalität zwischen Einfach- und Mehrfachfallbeschreibungen, möglicherweise aufgrund einer Kombination von Selektions- und Publikationsbias. Die Unterschätzung der tatsächlichen Mortalitätsrate kann zu unangebracht intensiven Therapieansätzen führen. Kliniker und Ethiker sollten daher vorsichtig sein, ihre Entscheidungen bei seltenen oder neuartigen Erkrankungen auf kumulative Erfahrungen von Fallbeschreibungen, insbesondere von Einzelfallbeschreibungen, zu basieren, die positive Verläufe von Behandlungen $\mathrm{zu}$ betonen scheinen.

Schlüsselwörter Epidermolysis bullosa $\cdot$ Prognose $\cdot$ Pylorusatresie $\cdot$ Mortalität · Klinische Ethik

\title{
Aspects associated with clinical decision-making based on case reports-ethical implications based on the example of a patient with Carmi syndrome
}

\begin{abstract}
Aim In case of extremely rare diseases, case reports are often the only experience to draw from for evidence-based management. Carmi syndrome is a rare, mostly lethal combination of junctional epidermolysis bullosa and pyloric atresia. During an ethical board, there were differences in perception of mortality rate. We tested the hypothesis that the cumulative mortality of single case reports is lower than that of multiple case series.

Case A baby girl was born at 33 weeks gestation with Carmi syndrome. The treatment options discussed in an interdisciplinary ethics board were a palliative approach versus surgical gastrojejunostomy. Because about one third of operated children described in case reports survived, we opted for surgical treatment. The patient died a painful death 4 weeks later.

Methods The PubMed database was systematically searched for reports of Carmi syndrome. Single case reports were compared to case series in terms of outcome.

Results A total of 102 cases of Carmi syndrome were identified in the literature. Mortality of single case reports was 17 out of 27 patients $(63 \%)$, while that of case series was higher at 62 out of 74 patients $(84 \%, p=0.036)$.

Conclusions Selection and publication bias may lead to inflation of survival rates in single case reports because successful cases are more likely to be published in the literature. These biases may lead to inappropriately aggressive treatment in futile cases. Clinicians should be cautious when discussing prognosis and making decisions based on the cumulative experience of case reports of extremely rare or novel diseases.
\end{abstract}

Keywords Epidermolysis bullosa $\cdot$ Prognosis $\cdot$ Pyloric atresia $\cdot$ Case series · Mortality 


\section{Einleitung}

Nach der Prämisse einer modernen, wissenschaftsorientierten Medizin sollen Kliniker ihre Entscheidungen aus der besten erhältlichen Evidenz ableiten. Die Basis dazu liefert die medizinische Fachliteratur. Gerade bei extrem seltenen oder neuartigen Erkrankungen ist ein solches Vorgehen jedoch schwierig, denn zum einen ist quantitativ oft nur wenig Information über die betreffende Krankheit erhältlich, zum anderen ist die Qualität der erhältlichen Studien aufgrund der niedrigen Fallzahlen eingeschränkt. In manchen Fällen sind lediglich Fallbeschreibungen publiziert. Allerdings kann sich deren Interpretation zur Erstellung von Therapiekonzepten aufgrund von Mechanismen systematischer Verzerrung als klinisch und ethisch problematisch erweisen, wie hier anhand eines Beispiels, das möglicherweise auf ein generelles Problem hindeutet, erläutert werden soll.

\section{Das Carmi Syndrom}

Das Carmi Syndrom ist eine extrem seltene, meistens letale Kombination von junktionaler Epidermolysis bullosa und Pylorusatresie (Pfendner und Lucky 2017). Insgesamt wurden bislang in der medizinischen Literatur nur knapp über 100 Fälle beschrieben. Die Erkrankung wird autosomal rezessiv vererbt und ist damit häufiger bei konsanguinen Eltern. Aufgrund der Pylorusatresie, also des angeborenen Verschlusses des Magenpförtners, können die betroffenen Patienten ohne chirurgischen Eingriff nicht enteral ernährt werden. Sie verdursten oder verhungern, falls ihnen keine intravenöse Flüssigkeit oder intravenöse Ernährung zugeführt wird. Die Epidermolysis bullosa führt zur Ablösung der Haut im gesamten Körper, so dass Oberflächenkeime in den Organismus eindringen und zur Sepsis führen können, die meist auch die Todesursache darstellt.

Ursächlich für die Erkrankung ist ein Gendefekt im ITGA6, ITGB4 oder im PLEC Gen (Pfendner und Lucky 2017). Die Diagnose wird heute genetisch gesichert, wobei derzeit auch die elektronenmikroskopische Analyse einer Hautbiopsie oder eine immunhistochemische Färbung zur Anwendung kommt.

Es gibt keine ursächliche Behandlung des Carmi Syndroms. Chirurgisch kann durch eine Verbindung zwischen Magen und Zwölffingerdarm, eine sogenannte Gastroduodenostomie, die Kontinuität des Verdauungstraktes hergestellt werden. AuBerdem kann durch Abtragen der abgelederten Haut und durch die anschließende Auflage von speziellen Hautersatzmaterialien eine temporäre Deckung der betroffenen Körperareale erreicht werden.

\section{Fallbeschreibungen in der evidenzbasierten Medizin}

Fallbeschreibungen (case reports) werden neben der Expertenmeinung vom Oxford Centre for Evidence-based Medicine als die hierarchisch schwächste Informationsquelle in der Evidenzbasierten Medizin angesehen (Oxford Center for EBM 2009). Dabei nehmen sie jedoch für sich betrachtet eine wichtige Rolle in der Medizin ein. Ohne Fallbeschreibungen hätten sich bestimmte chirurgische Techniken nicht verbreitet, die teratogenen Eigenschaften von Thalidomid (Contergan $\left.{ }^{\circledR}\right)$ wären erst viel 
später erkannt worden, und eine rätselhafte Immunschwäche, die sich Anfang der 1980er Jahre ausbreitete, wäre ohne Beschreibung von Indexfällen nicht so schnell als Infektion mit dem Humanen Immundefizienz-Virus (HIV) identifiziert worden. Fallbeschreibungen haben demnach in der Medizin durchaus ihren Stellenwert, insbesondere bei neuen oder sehr seltenen Erkrankungen sowie zur Beschreibung von innovativen therapeutischen Ansätzen. Allerdings wurden von anderen bereits in der Vergangenheit die Limitationen der Ableitung von Therapieeffekten anhand von kollektiven Fallbeschreibungen erörtert (Caster et al. 2014).

\section{Zielsetzung}

In dieser Arbeit sollen anhand eines Falles von Carmi Syndrom die ethischen Probleme aufgezeichnet werden, die dabei entstehen können, wenn klinische Entscheidungen anhand einer Sammlung von einzelnen publizierten Fallbeschreibungen getroffen werden. Dabei sollen insbesondere die Probleme des Under- und Overreporting sowie die Phänomene des Selektions- und Reportingbias diskutiert werden. Unsere Hypothese ist, dass Fälle mit erfolgreichem Outcome häufiger als einzelne Fallbeschreibungen publiziert werden und damit zu einer fälschlicherweise subjektiv höheren angenommenen Überlebensrate führen, die wiederum die klinischen Entscheidungsprozesse bis hin zu ihrer normativen Begründung bei aussichtslosen Fällen beeinflussen kann.

\section{Klinische Fallvorstellung}

Die Patientin wurde in der 33. Schwangerschaftswoche per Notfallsektio bei drohender Plazentalösung in unserer Klinik geboren. Die Mutter war eine mit dem Vater konsanguine Frau, die bereits 5 Spontanaborte erlitten hatte. Von 6 Lebendgeburten waren 2 Mädchen unmittelbar postpartal verstorben. Die APGAR Werte (Score zur klinischen Einschätzung von Neugeborenen von 0 [minimal] bis 10 [maximal] aufgrund von Atmung, Puls, Grundtonus, Aussehen und Reflexe) waren 8 bei einer Minute, 8 bei $5 \mathrm{~min}$. Nach der Geburt erhielt die Patientin empirisch eine antibiotische Behandlung mit Ampicilllin und Gentamicin.

Bei der körperlichen Untersuchung fielen Ablederungen der Haut am gesamten Körper einschließlich des Gesichts und der Extremitäten auf. Das initiale Röntgenbabygramm zeigte eine solitäre Luftblase im linken oberen Quadranten des Abdomens, vereinbar mit einer Pylorusatresie. Anhand der Kombination von Hautablederung im Sinne einer Epidermolysis bullosa und der Pylorusatresie wurde die Diagnose des Carmi Syndroms gestellt. Zur Bestätigung wurde eine Hautbiopsie entnommen und eine genetische Analyse durchgeführt.

\section{Perspektiven der Teilnehmer der Ethikberatung}

Aufgrund der komplexen medizinischen und psychosozialen Situation, die sowohl für die Eltern als auch das Team als belastend empfunden wurde, wurde das klinische 
Ethikkomitee zu einer Ethikberatung einberufen. Dabei waren neben zwei Vertretern des Klinischen Ethikkomitees die behandelnden Ärzte aus der Neonatologie und der Kinderchirurgie anwesend. Die Eltern wurden separat über einen Dolmetscher eingebunden. Aufgrund der Latenz der Untersuchungen lagen die genetischen und die immunhistochemischen Ergebnisse zur Zeit der Ethikberatung noch nicht vor.

\section{Neonatologische Perspektive}

Aus neonatologischer Sicht war die schlechte Prognose zwar augenscheinlich, jedoch wurden eine Reihe von Fallbeschreibungen diskutiert, in denen ein langfristiges Überleben der betroffenen Patienten dokumentiert worden war. Um eine enterale Ernährung zu ermöglichen, wurde daher die chirurgische Intervention zur Herstellung der gastrointestinalen Kontinuität erbeten. Eine wesentliche Rationale für dieses Vorgehen war dabei der Wunsch der Eltern nach maximaler Therapie. Um Leiden zu minimieren, wurde eine großzügige analgetische Therapie mit Opiaten als indiziert angesehen.

\section{Kinderchirurgische Perspektive}

Der initiale Vorschlag aus Sicht der beteiligten Kinderchirurgen war es, von einer operativen Intervention abzusehen und das Kind palliativ zu behandeln. Diese Sichtweise basierte auf einer Vielzahl von weiteren Fallbeschreibungen, in denen die Mehrzahl der betroffenen Patienten nach Eingriffen letztlich verstarben. Zudem war davon auszugehen, dass die mechanische Manipulation der Bauchdecke durch Retraktion bei einer offenen Operation zu Ablederung der betroffenen Hautareale führen würde und die Situation dadurch verschlechtert werden könnte. Wenn überhaupt, sei daher ein minimalinvasives, laparoskopisches Verfahren zu favorisieren. Als Vorteil einer Operation wurde allerdings auch gesehen, dass gleichzeitig ein getunnelter, langfristiger zentralvenöser Katheter angelegt und die Abdeckung der offenen Wunden durch eine Hautersatzmembran in einer Narkose erfolgen könnten.

\section{Perspektive der Eltern}

Die Eltern verbalisierten die Erwartung, dass ,alles für ihre Tochter getan werde“. Dies begründeten sie mit ihrem kulturellen und religiösen Hintergrund. Auf Nachfragen, ob hiermit auch ein chirurgischer Eingriff gemeint wäre, bejahten sie dies. Es erfolgte die dezidierte Aufklärung über Vor- und Nachteile einer Kontinuitätsherstellung des Pylorus, einer Gastrostomie (Ernährungssonde in den Magen), und einer Versorgung der abgelederten Hautareale. Insbesondere wurde erklärt, dass die Grunderkrankung nicht heilbar sei und trotz operativem Eingriff mit einem letalen Ausgang zu rechnen sei. Die Eltern berieten sich und gaben schließlich ihr schriftliches Einverständnis für den Eingriff, mit der Bitte, ihre Tochter möglichst bald zu operieren. 


\section{Perspektive der Klinischen Ethik}

Die klinisch-ethische Einschätzung des Falles hatte zunächst zum Ausgangspunkt, dass es sich bei dem Mädchen nicht um ein im Sterbeprozess befindliches Kind handelte. In einer Entscheidung für oder gegen eine Eskalation der Therapie bis hin zur chirurgischen Intervention stand zunächst die Frage im Mittelpunkt, was als das beste Interesse (best interest) des Kindes angesehen werden könne. Insbesondere die Tatsache, dass der Therapieerfolg unsicher erschien, sowie die Frage, ob eine invasive Therapie für das Kind so gestaltet werden kann, dass diese nicht zu unnötigem zusätzlichen Leid führt, waren in der Abwägung zentral. Begleitet durch eine adäquate Schmerztherapie erschien die Herstellung der Ernährungsfähigkeit auf enteralem Wege im besten Interesse des Kindes zu sein und so der Wunsch der Eltern als ethisch rechtfertigbar.

\section{Outcome des Falles}

Immunhistochemisch fand sich ein komplettes Fehlen des Adhäsionsmoleküls $\alpha 6 \beta 4$ Integrin in der Haut. Damit vereinbar ergab die genetische Analyse eine homozygote Mutation im ITGA6 Gen und damit eine eindeutige Diagnose des Carmi Snydroms.

Am 8. Lebenstag wurde auf der Grundlage des Ethikkonsils eine laparoskopische Gastrojejunostomie und Gastrostomie durchgeführt. Die abgelederten Hautareale wurden mit einer Hautersatzmembran aus Polylactid, Trimethylencarbonat und $\varepsilon$-Caprolacton (Suprathel ${ }^{\circledR}$, PolyMedics Innovations GmbH, Denkendorf, BRD) abgedeckt. In derselben Operation wurde außerdem ein getunnelter, zentralvenöser Broviac-Katheter angelegt.

Trotz der nun hergestellten Durchgängigkeit des Gastrointestinaltraktes gestaltete sich der Kostaufbau aufgrund einer Motilitätsstörung als schwierig, so dass die Patientin hauptsächlich parenteral über einen zentralvenösen Katheter ernährt wurde.

Aufgrund der Ablösung der Schleimhäute kam es immer wieder zu einer Verlegung der Atemwege. Außerdem entwickelte die Patientin Sepsis mit Staphylococcus epidermidis. Sie verstarb an den Folgen der Infektion und der Atemwegsbehinderung im Alter von 35 Tagen.

\section{Wissenschaftliche Fragestellung und Analyse der Literatur zum Carmi Syndrom}

In der Ethikberatung ergaben sich Unterschiede in der Wertung der in der Literatur angegebenen Mortalität bei Carmi Syndrom. Da diese Entität extrem selten ist, existieren bislang nur Fallbeschreibungen und kleine Fallserien. Um zu verstehen, wie die unterschiedlichen Einschätzungen auf der Basis der vorhandenen Literatur entstehen könnten, wäre nun die Hypothese zu prüfen, ob ein Publikationsbias bestehen könnte, aufgrund dessen die Überlebensrate höher erscheint, als sie tatsächlich ist. Dies könnte darauf beruhen, dass Fälle mit positivem Outcome häufiger publiziert werden, entweder weil die Autoren eine größere Motivation empfinden, solche Fälle 
in einem Manuskript zusammenzuschreiben, oder weil Gutachter und Herausgeber von Fachzeitschriften eher dazu tendieren, positive Fallberichte zur Publikation anzunehmen und negative abzulehnen. Im letzteren Fall ergäben Fallserien (also Publikationen, die zwei oder mehr Fälle beschreiben) ein objektiveres Bild über die tatsächliche Mortalität der Erkrankung, weil Autoren eventuell dazu tendieren würden, bei einer größeren Erfahrung mit der Erkrankung auch die Fälle zu erwähnen, die nicht überlebt haben. Unsere Hypothese ist demnach, dass auch bei der hier diskutierten klinischen und ethischen Entscheidung die niedrigere publizierte Mortalität bei Einzelfallbeschreibungen (und damit die höhere Überlebensrate) im Vergleich zu Mehrfachfallbeschreibungen zu einer falsch positiven Bewertung geführt haben könnte.

Tab. 1 Übersicht der Einzelfallbeschreibungen von Carmi Syndrom in der Literatur bis einschließlich $2019(n=27)$

\begin{tabular}{|c|c|c|c|}
\hline Erstautor & Erscheinungsjahr & Fälle & Davon überlebt \\
\hline Hicks et al. (2018) & 2018 & 1 & 0 \\
\hline Trah et al. (2018) & 2018 & 1 & 0 \\
\hline Hattori et al. (2018) & 2018 & 1 & 1 \\
\hline Singh et al. (2017) & 2017 & 1 & 0 \\
\hline Kayki et al. (2017) & 2017 & 1 & 1 \\
\hline Masunaga et al. (2017) & 2017 & 1 & 0 \\
\hline Mutlu et al. (2015) & 2015 & 1 & 0 \\
\hline Masunaga et al. (2015) & 2015 & 1 & 1 \\
\hline Hon et al. (2015) & 2015 & 1 & 0 \\
\hline Marjanovic et al. (2013) & 2013 & 1 & 0 \\
\hline Merrow et al. (2013) & 2013 & 1 & 0 \\
\hline Maurice et al. (2013) & 2013 & 1 & 0 \\
\hline Joshi et al. (2012) & 2012 & 1 & 1 \\
\hline Diociaiuti et al. (2013) & 2013 & 1 & 1 \\
\hline Kim et al. (2011) & 2011 & 1 & 1 \\
\hline Stoevesandt et al. (2012) & 2012 & 1 & 0 \\
\hline Aydin et al. (2010) & 2010 & 1 & 0 \\
\hline Natsuga et al. (2010) & 2010 & 1 & 0 \\
\hline Abe et al. (2007) & 2007 & 1 & 1 \\
\hline Sarin und Nagdeve (2006) & 2006 & 1 & 0 \\
\hline De Jenlis Sicot et al. (2005) & 2005 & 1 & 0 \\
\hline Micheloni et al. (2004) & 2004 & 1 & 0 \\
\hline Allegra et al. (2003) & 2003 & 1 & 0 \\
\hline Chavanas et al. (1999) & 1999 & 1 & 1 \\
\hline Ha et al. (1998) & 1998 & 1 & 1 \\
\hline Pulkkinen et al. (1998a) & $1998 \mathrm{a}$ & 1 & 1 \\
\hline \multirow[t]{3}{*}{ Ruzzi et al. (1997) } & 1997 & 1 & 0 \\
\hline & Kumulativ & 27 & 10 \\
\hline & Überlebensrate & - & $37 \%$ \\
\hline
\end{tabular}


Tab. 2 Übersicht der Mehrfachfallbeschreibungen von Carmi Syndrom in der Literatur bis einschließlich $2019(n=17)$

\begin{tabular}{|c|c|c|c|}
\hline Erstautor & Erscheinungsjahr & Fälle & Davon überlebt \\
\hline Wang et al. (2019) & 2019 & 3 & 0 \\
\hline Mencía et al. (2016) & 2016 & 6 & 2 \\
\hline Bıçakcı et al. (2012) & 2012 & 2 & 0 \\
\hline Ozge et al. (2012) & 2012 & 3 & 0 \\
\hline Chung und Uitto (2010) & 2010 & 2 & 0 \\
\hline Frew et al. (2010) & 2010 & 7 & 3 \\
\hline Salvestrini et al. (2008) & 2008 & 2 & 1 \\
\hline Dang et al. (2008) & 2008 & 3 & 1 \\
\hline Birnbaum et al. (2008) & 2008 & 13 & 0 \\
\hline Pfendner und Uitto (2005) & 2005 & 6 & 0 \\
\hline Iacovacci et al. (2003) & 2003 & 6 & 0 \\
\hline Ashton et al. (2001) & 2001 & 2 & 0 \\
\hline Nakano et al. (2001) & 2001 & 7 & 3 \\
\hline Dural et al. (2001) & 2001 & 3 & 0 \\
\hline Pulkkinen et al. (1998b) & $1998 b$ & 3 & 0 \\
\hline Pulkkinen et al. (1998c) & $1998 \mathrm{c}$ & 5 & 3 \\
\hline \multirow[t]{6}{*}{ Takizawa et al. (1997) } & 1997 & 2 & 0 \\
\hline & Kumulativ & 75 & 13 \\
\hline & Spannweite & $2-13$ & - \\
\hline & Median & 3 & - \\
\hline & Mittelwert \pm Standardabweichung & $4,5 \pm 2,9$ & - \\
\hline & Überlebensrate & - & $17 \%$ \\
\hline
\end{tabular}

Tab. 3 Pearson Chi-Quadrat Test des Parameters „Überleben“ beim kumulativen Vergleich von Einzelversus Mehrfachbeschreibungen (Uitenbroek 2019)

\begin{tabular}{llll}
\hline & Lebend & Tot & Gesamt \\
\hline Einzelfallbeschreibungen & 10 & 17 & 27 \\
Mehrfachfallbeschreibungen & 13 & 62 & 75 \\
Gesamt & 23 & 79 & 102 \\
& & & $p=0,036$ \\
\hline
\end{tabular}

\section{Methodik}

Um die Hypothese zu prüfen, ob sich die kumulativ publizierte Überlebenswahrscheinlichkeit der Einzel- beziehungsweise Mehrfachfallberichte unterscheidet, wurden alle in PubMed publizierten Arbeiten zum Carmi Syndrom mit Angaben zum Outcome analysiert. Dazu wurde im Dezember 2019 eine Recherche unter www. pubmed.gov unter dem Stichwort <Carmi Syndrome> (59 Titel) und unter der Kombination <pyloric atresia AND epidermolysis bullosa> (184 Ergebnisse) unternommen. Um in die Studie eingeschlossen zu werden, mussten die Publikationen gesicherte Fälle von Carmi Syndrom und das Outcome der Patienten (tot/lebend) beschreiben. Doppelte Publikationen von identischen Fällen wurden nur einmal be- 
rücksichtigt. Die betreffenden Artikel wurden nach Einzel- beziehungsweise Mehrfachfallbeschreibungen geordnet und die Ergebnisse mit dem Pearson Chi-Quadrat Test verglichen. Ein $p$ von $<0,05$ wurde als signifikant definiert.

\section{Ergebnisse der Literaturanalyse}

Die Recherche nach <Carmi Syndrome > ergab 59, die nach < pyloric atresia AND epidermolysis bullosa $>184$ Titel. Relevant erschienen 129 Titel, von denen die Abstracts gescreent wurden. Von diesen wurden 62 Volltextartikel heruntergeladen. Dabei fanden sich insgesamt 102 Fälle von Carmi Syndrom aus 27 Einzelfallbeschreibungen (Tab. 1) und 17 Mehrfachfallbeschreibungen (Tab. 2), die die Einschlusskriterien erfüllten und in der Analyse berücksichtigt wurden. Bei den Einzelfallbeschreibungen fand sich eine signifikant höhere gepoolte Überlebenswahrscheinlichkeit von $37 \%$ als bei den Mehrfachfallbeschreibungen (17\%, $p=0,036$, siehe Tab. 3).

\section{Diskussion}

Die Ethikberatung bei extrem seltenen Erkrankungen stellt für alle Beteiligten eine besondere Herausforderung dar. Die eingeschränkte oder fehlende Erfahrung mit Prognose und Verlauf führt zu Unsicherheit bei der Einschätzung von Chancen und Risiken von therapeutischen Handlungen und bei Kindern daher zu erheblichen Unschärfen der Einschätzung, welches Vorgehen im besten Interesse der Patienten ist. Nicht selten kommt es dabei zu Situationen, in denen nicht mehr klar zu entscheiden ist, ob sich das Leiden eines Kindes vorrangig aus der Grunderkrankung oder aus den Folgen der - teils hoch invasiven - Behandlungen ergibt. Bei extrem seltenen Erkrankungen müssen sich die Kliniker meist auf Fallbeschreibungen und Fallserien stützen. Diese sind allerdings augenscheinlich besonders anfällig für bestimmte systematische Verzerrungen wie dem Publikationsbias.

\section{Medizinischer Hintergrund zum Carmi Syndrom}

Das Carmi Syndrom ist eine dieser extrem seltenen Erkrankungen, die im hier vorgestellten Fall solche systematischen Verzerrungen zunächst nur vermuten ließ. Das bislang größte systematische Review wurde erst kürzlich von Mylonas et al. (2019) publiziert. Von den 100 eingeschlossenen Patienten waren insgesamt 70 verstorben. Eine Operation wurde an 73 Patienten durchgeführt, von denen 49 verstarben $(67 \%)$. Eine gesonderte Unterscheidung der Mortalität nach Einfach- und Mehrfachfallbeschreibungen wurde nicht durchgeführt. Besonders ominöse prädiktive Faktoren waren dabei die fehlende oder verminderte Expression von Integrin $\alpha 6 \beta 4$ sowie Mutationen im Integrin $\alpha 6$, Plectin- 1 und im cephalen Integrin $\beta 4$. Viele Fälle sind mit anderen Malformationen, insbesondere des Gastrointestinaltraktes und des Urogenitaltraktes assoziiert. Über die Hälfte der beschriebenen Patienten waren Frühgeborene (Mylonas et al. 2019). 


\section{Ethischer Hintergrund}

Im Rahmen der Ethikberatung unseres Falles ergab sich der Konflikt, ob primär ein chirurgisch-therapeutischer oder ein palliativer Ansatz angebracht gewesen wäre. Die unterschiedliche Sichtweise ergab sich aus der klassischen Überlegung, ob das Glas halbvoll oder halbleer sei. In der Literatur gab es Fallbeschreibungen, die beim Carmi Syndrom sowohl ein langfristiges Überleben, aber auch ein qualvolles Sterben der Betroffenen skizzierten. Ein möglicher Ansatz wäre gewesen, auf die genetische und immunhistochemische Untersuchung zu warten, da hierbei das komplette Fehlen von Integrin $\alpha 6 \beta 4$ bei unserer Patientin eine infauste Prognose aufgezeichnet hätte. In diesem Zusammenhang wäre das Einhalten von vorher definierten STOP-Kriterien für eine invasive Therapie sinnvoll, bis alle prognostischen Marker für eine objektive Einschätzung verfügbar wären. $\mathrm{Zu}$ bedenken ist dabei freilich auch, dass ein sich daraus möglicherweise ergebendes ,therapeutisches Moratorium“ nachteilig auf die Prognose oder den Therapieerfolg auswirken kann, sollte bis zum Vorliegen aller relevanten Befunde zu viel Zeit vergehen. Dieser Umstand ist vor allem bei komplexeren molekulargenetischen Untersuchungen nicht selten und auch der eindeutige molekulare Befund vermittelt nicht immer die für eine medizinische und ethische Bewertung von Therapiezielen erforderliche Sicherheit (Kidszun et al. 2016). So entschied sich das Team schließlich aufgrund der sich verschlimmernden Ablederungen der Haut, dem Drängen der Eltern nach einem operativen Eingriff und der Notwendigkeit eines zentralvenösen Zugangs für eine zeitnahe chirurgische Intervention. Retrospektiv hat die Patientin nicht in erwünschter Weise davon profitiert. Im Gegenteil, das Leiden der Patientin wurde dadurch eher verlängert.

\section{Vor- und Nachteile von Fallbeschreibungen}

Fallbeschreibungen und Fallserien können allgemein als Beobachtungsstudien (observational studies) kategorisiert werden. Ein grundsätzlicher Anspruch an eine solche Publikation ist die Beschreibung einer neuen, nicht bekannten Erkrankung, Beobachtung oder Therapie, oder eines anderen außergewöhnlichen Aspektes (Shevell 2004). Dabei steht im Vordergrund die qualitative, nicht die quantitative Analyse. Folglich sind Beobachtungsstudien nicht geeignet, statistische Parameter wie Effektgröße, Inzidenz, Prävalenz oder Mortalitätsrate zu beschreiben. Um die Qualität von Fallbeschreibungen und Fallserienbeschreibungen in der Chirurgie zu optimieren und zu standardisieren, wurden entsprechende SCARE (consensus on Surgical CAse REport) und PROCESS (Preferred Reporting Of CasE Series in Surgery) Leitlinien publiziert (Agha et al. 2018a, b). Aus ethischer Sicht sind die Einwilligung der Patienten oder deren Betreuer zur Publikation des Falles (informed consent to publish) und die vertrauliche Behandlung von persönlicher Information (confidentiality) Mindestanforderungen (Shevell 2004). Der Übergang von Fallbeschreibung (Case report) zu Fallserie (Case series) ist fließend, manche Autoren definieren Fallserien als Beschreibung von mehr als vier Fällen (Abu-Zidan et al. 2012). Manche Universitätsklinika fordern zur Publikation von Fallserien ab einer bestimmten Anzahl von Patienten (beispielsweise drei) ein Votum der Ethikkommission (Boston University 2019). 
Die Probleme und Limitationen von Fallbeschreibungen wurden bereits in der Literatur diskutiert (Nissen und Wynn 2014; Kestenbaum 2009) und beinhalten 1. das Unvermögen, allgemeingültige Schlüsse zu ziehen, 2. das Fehlen einer Kontrollgruppe, 3. keine Aussage über die Zusammenhänge von Ursache und Wirkung, 5. die Stichprobenverzerrung, 6. die Gefahr der Überinterpretation, 7. Effekte aufgrund von Publikationsbias, 8. die retrospektive Methodik sowie 9. die Distraktion des Lesers durch besonderes Hervorheben des Ungewöhnlichen.

Relevant im Zusammenhang mit der Ethikberatung im beschriebenen Fall sind insbesondere die Aspekte des Publikationsbias und der Überinterpretation sowie deren deutliche Auswirkungen auf die aus ethischer Sicht ohnehin bereits prekäre Abwägung von Lebensschutz und Würdeschutz. Hierbei spielen zum einen quantitative Aspekte wie Mortalität und Morbidität im Hinblick auf mögliche Komplikationen eine Rolle, auf der anderen Seite haben aber gerade auch qualitative Aspekte, insbesondere die akute und langfristige Lebensqualität, ein spezifisches Gewicht in der ethischen Abwägung. Wenn langfristig eine gute oder akzeptable Lebensqualität erreicht werden kann, sind auch kurzfristige Belastungen als Opportunitätslasten im Sinne eines operativen Eingriffes, einer maschinellen Beatmung, oder einer ausgiebigen, komplexen, und nicht immer schmerzlosen Wundbehandlung medizinisch und ethisch geboten. In der Frage, wieviel Leid man einem Patienten zumuten kann, spielen aber auch vor allem Überlegungen eine Rolle, wie das Kindeswohl als leitendes Prinzip in konkreten Situationen zur Entfaltung gebracht werden kann (Inthorn und Paul 2017). Abgeleitet vom Konzept der Autonomie des Patienten ergibt sich die Frage, ob es grundsätzlich im Interesse des Kindes sein kann, das mit einer Therapieeskalation verbundene Leid in Aussicht auf einen - freilich mehr oder weniger - unsicheren Therapieerfolg auf sich zu nehmen. Das Problem mit diesem mutmaßlichen Interesse auch in dem hier berichteten Fall ist, dass die Patientin selbst aufgrund ihres Alters nicht einwilligungsfähig ist und den emotional in erheblicher Weise betroffenen Eltern die Verantwortung zukommt, über die Intensität und Dauer einer Therapie zu entscheiden, was nicht selten als normative Überlastung im Sinne eines ,moral distress“ wahrgenommen wird. Letztlich muss in eine solche durch Kliniker und Ethiker begleitete Entscheidung auch die Mortalität der Erkrankung vorbehaltlos mit in den Blick genommen werden. Ist sie insgesamt so hoch, dass nur in seltenen Ausnahmefällen ein Überleben erreicht werden kann, so ist ein würdevolles, palliativ begleitetes Sterben einem verlängerten, perspektivlosen Martyrium vorzuziehen. Insofern ist es durchaus ein Unterschied für die Wahrung der kindlichen Interessen, ob man von einer Überlebensrate von $17 \%$ oder $37 \%$ ausgehen kann und welche Vorbehalte sich aus der einen oder anderen Lesart ergeben mögen.

\section{Bedeutung von Fallberichten bei der Einschätzung von extrem seltenen oder neuartigen Erkrankungen}

Um über das hier geschilderte Problem im Zusammenhang mit dem Carmi Syndrom hinaus die klinische und ethische Relevanz von Fallberichten sowohl im Form von Fallreihen als auch in Einzelfallberichten besser zu durchdringen, lohnt sich ein systematischer Blick auf die Publikationslandschaft. Allgemein werden positive 
Fallbeschreibungen eher publiziert als negative (Easterbrook et al. 1991). In einer Studie dokumentierten weniger als $10 \%$ der über einen Zeitraum von 18 Monaten in der Zeitschrift Lancet publizierten Fallbeschreibungen ein Therapieversagen (Albrecht et al. 2005). Das Phänomen der Überinterpretation beinhaltet mitunter eine emotionale Komponente durch den Leser. So werden in einem auch als ,anecdotal falacy“ beschriebenen Mechanismus subjektive Hoffnungen auf ein bestimmtes Outcome aus einer Publikation heraus verallgemeinert (Charlton und Walston 1998). Fallbeschreibungen sind letztendlich Geschichten, die uns berühren wie die eigene, anekdotische Erfahrung. Etwas pointiert könnte man fragen, ob das Publizieren von positiven Fallbeschreibungen nicht gegen das Wahrhaftigkeitsgebot verstößt, indem ungünstige Ergebnisse unterdrückt werden. In den Publikationsleitlinien zu Fallbeschreibungen könnte von Herausgebern generell gefordert werden, dass die Autoren immer auch nach vergleichbaren Fällen mit negativem Outcome in ihrer Institution suchen und diese in der Publikation miterwähnen müssen, um dem Problem des kleinen Nenners wenigstens ansatzweise zu begegnen.

In unserer Analyse der Literatur zum Carmi Syndrom war die Gesamtmortalität in den Mehrfachbeschreibungen um etwa ein Drittel höher als in den Einzelfallbeschreibungen, die Überlebensrate weniger als halb so hoch. Dies lässt darauf schließen, dass durch einen Selektionsmechanismus die Überlebensrate bei Einzelfallbeschreibungen falsch positiv erhöht erscheint. Ferner könnte man annehmen, dass dieser Mechanismus umso deutlicher ausgeprägt ist, je seltener die Krankheit auftritt und je weniger Fallserien (Mehrfachfallbeschreibungen) dazu existieren. Wir schlagen daher zur besseren Einordnung dieser Verzerrung einen entsprechenden Index in Bezug auf das Outcome von Einzel- zu Mehrfachfallbeschreibungen vor. In unserem Falle beliefe sich ein solcher als SMI (Single to Multiple casereport Index) definierter Wert für den Parameter Überlebensrate bei Carmi Syndrom auf $37 \%$ dividiert durch $17 \%$, was 2,18 entspräche. Unseres Wissens wurde ein derartiger Index als quantitatives Instrument zur Beschreibung des Publikationsbias im Zusammenhang mit extrem seltenen Erkrankungen noch nicht beschrieben.

Der SMI könnte so bei der Betrachtung von Fallbeschreibungen im Rahmen einer Ethikberatung darüber Aufschluss geben, ob eine einzelfallbasierte Inflation der Überlebensrate vorliegen könne. Je höher der Index, um so höher der potentielle Effekt. Dadurch könnte man das Vorliegen einer infausten Prognose eventuell besser einschätzen und so die Entscheidung zu einem palliativen Vorgehen möglicherweise objektiver begründen.

\section{Limitationen der Studie}

Unsere Studie beinhaltet freilich einige Limitationen. Auch für diese Studie gilt, dass Literatur zum Carmi Syndrom analysiert wurde und die Übertragbarkeit unserer Ergebnisse auf andere seltene oder gar neuartige Erkrankungen noch zu überprüfen wäre. Auch die untersuchte Literatur selbst brachte Einschränkungen mit sich. Zum einen war das Outcome nicht bei allen Patienten ersichtlich. Bei Publikationen, in denen nur ein Teil der Patienten mit Outcome beschrieben sind (zum Beispiel in drei von sechs Fällen bei Wang et al. (2019)) wurden nur die bekannten Fälle in die Analyse eingeschlossen. Außerdem wurde nicht bei allen Publikationen der Zeitraum 
der Verlaufsbeobachtung, also des Follow-up, dokumentiert. Es kann also durchaus sein, dass die Mortalität noch höher ist, da Patienten, die bei der Publikation noch lebten, danach an den Folgen der Krankheit verstorben sein könnten.

\section{Zusammenfassung und Fazit}

Zusammenfassend verdeutlicht der hier beschriebene Fall und die vorliegende Diskussion, dass bei der Interpretation von Fallbeschreibungen zur Bestimmung der Prognose von extrem seltenen Erkrankungen sowohl hinsichtlich der klinischen Bedeutung als auch in Bezug auf die Bedeutung der Befunde für das beste Interesse von nicht-einwilligungsfähigen Patienten oder aber die Bereitschaft zum Tragen von Opportunitätslasten bei einwilligungsfähigen Patienten Vorsicht geboten ist. Dies beinhaltet auch, dass Effekte systematischer Verzerrung im Rahmen von Ethikberatungen bekannt sein und erkannt werden müssen. Nicht selten ist die ethische Analyse im Rahmen situativen Entscheidens von der Bonität der klinischen Information sowie der Evidenzlage abhängig. Für eine unabhängige klinisch-ethische Analyse ist es daher gerade wenn es um seltene oder neuartige Erkrankungen geht unerlässlich, sich nicht auf ad hoc angebotene Interpretationen der Fachliteratur zu verlassen, sondern im besten Sinne der Evidenzbasiertheit einen kritisch-hermeneutischen Zugang zu der für eine Beratung erforderlichen Wissensbasis zu erarbeiten. Eine Überinterpretation von positiven Ergebnissen kann zur Anwendung oder Aufrechterhaltung einer unverhältnismäßig aggressiven, aussichtslosen und damit den Interessen des Patienten entgegenstehenden, ethisch problematischen Therapie führen.

Funding Open Access funding provided by Projekt DEAL.

Open Access Dieser Artikel wird unter der Creative Commons Namensnennung 4.0 International Lizenz veröffentlicht, welche die Nutzung, Vervielfältigung, Bearbeitung, Verbreitung und Wiedergabe in jeglichem Medium und Format erlaubt, sofern Sie den/die ursprünglichen Autor(en) und die Quelle ordnungsgemäß nennen, einen Link zur Creative Commons Lizenz beifügen und angeben, ob Änderungen vorgenommen wurden.

Die in diesem Artikel enthaltenen Bilder und sonstiges Drittmaterial unterliegen ebenfalls der genannten Creative Commons Lizenz, sofern sich aus der Abbildungslegende nichts anderes ergibt. Sofern das betreffende Material nicht unter der genannten Creative Commons Lizenz steht und die betreffende Handlung nicht nach gesetzlichen Vorschriften erlaubt ist, ist für die oben aufgeführten Weiterverwendungen des Materials die Einwilligung des jeweiligen Rechteinhabers einzuholen.

Weitere Details zur Lizenz entnehmen Sie bitte der Lizenzinformation auf http://creativecommons.org/ licenses/by/4.0/deed.de.

\section{Einhaltung ethischer Richtlinien}

Interessenkonflikt O. J. Muensterer und N. W. Paul geben an, dass kein Interessenkonflikt besteht.

Ethische Standards Für diesen Beitrag wurden von den Autoren keine Studien an Menschen oder Tieren durchgeführt. Für die aufgeführten Studien gelten die jeweils dort angegebenen ethischen Richtlinien. 


\section{Literatur}

Abe M, Sawamura D, Goto M, Nakamura H, Nagasaki A, Nomura Y, Kawasaki H, Isogai R, Shimizu H (2007) ITGB4 missense mutation in a transmembrane domain causes non-lethal variant of junctional epidermolysis bullosa with pyloric atresia. J Dermatol Sci 47:165-167

Abu-Zidan FM, Abbas AK, Hefny AF (2012) Clinical "case series": a concept analysis. Afr Health Sci 12:557-562

Agha RA, Borrelli MR, Farwana R, Koshy K, Fowler A, Orgill DP, For the SCARE Group (2018a) The SCARE 2018 statement: updating consensus Surgical CAse REport (SCARE) guidelines. Int J Surg 60:132-136

Agha RA, Borrelli MR, Farwana R, Koshy K, Fowler A, Orgill DP, SCARE Group (2018b) The PROCESS 2018 statement: updating consensus Preferred Reporting Of CasE Series in Surgery (PROCESS) guidelines. Int J Surg 60:279-282

Albrecht J, Meves A, Bigby M (2005) Case reports and case series from Lancet had significant impact on medical literature. J Clin Epidemiol 58:1227-1232

Allegra M, Gagnoux-Palacios L, Gache Y, Roques S, Lestringant G, Ortonne JP, Meneguzzi G (2003) Rapid decay of alpha6 integrin caused by a mis-sense mutation in the propeller domain results in severe junctional epidermolysis bullosa with pyloric atresia. J Invest Dermatol 121:1336-1343

Ashton GH, Sorelli P, Mellerio JE, Keane FM, Eady RA, McGrath JA (2001) Alpha 6 beta 4 integrin abnormalities in junctional epidermolysis bullosa with pyloric atresia. Br J Dermatol 144:408-414

Aydin M, Zenciroglu A, Yaman A, Orun UA, Arda N, Colak AG, Okumus N, Ipek MS, Ceylaner S (2010) Carmi syndrome with congenital heart defects. Am J Med Genet A 152A(8):2120-2122

Birnbaum RY, Landau D, Elbedour K, Ofir R, Birk OS, Carmi R (2008) Deletion of the first pair of fibronectin type III repeats of the integrin beta- 4 gene is associated with epidermolysis bullosa, pyloric atresia and aplasia cutis congenita in the original Carmi syndrome patients. Am J Med Genet A 146A(8):1063-1066

Boston University Medical Campus and Boston Medical Center Institutional Review Board (2019) Case reports and case series. http://www.bumc.bu.edu/irb/submission-requirements/special-submissionrequirements/case-reports-and-case-series/. Zugegriffen: 24. Apr. 2020

Bıçakcı U, Tander B, Cakmak Çelik F, Arıtürk E, Rızalar R (2012) Pyloric atresia associated with epidermolysis bullosa: report of two cases and review of the literature. Ulus Travma Acil Cerrahi Derg $18: 271-273$

Caster O, Noren GN, Edwards IR (2014) Computing limits on medicine risks based on collections of individual case reports. Theor Biol Med Model 11:15

Charlton BG, Walston F (1998) Individual case studies in clinical research. J Eval Clin Practice 4:147-155

Chavanas S, Gache Y, Vailly J, Kanitakis J, Pulkkinen L, Uitto J, Ortonne J, Meneguzzi G (1999) Splicing modulation of integrin beta4 pre-mRNA carrying a branch point mutation underlies epidermolysis bullosa with pyloric atresia undergoing spontaneous amelioration with ageing. Hum Mol Genet 8:2097-2105

Chung HJ, Uitto J (2010) Epidermolysis bullosa with pyloric atresia. Dermatol Clin 28:43-54

Dang N, Klingberg S, Rubin AI, Edwards M, Borelli S, Relic J, Marr P, Tran K, Turner A, Smith N, Murrell DF (2008) Differential expression of pyloric atresia in junctional epidermolysis bullosa with ITGB4 mutations suggests that pyloric atresia is due to factors other than the mutations and not predictive of a poor outcome: three novel mutations and a review of the literature. Acta Derm Venereol 88:438-448

De Jenlis Sicot B, Deruelle P, Kacet N, Vaillant C, Subtil D (2005) Prenatal findings in epidermolysis bullosa with pyloric atresia in a family not known to be at risk. Ultrasound Obstet Gynecol 25:607-609

Diociaiuti A, Castiglia D, Morini F, Boldrini R, Fortugno P, Zambruno G, El Hachem M (2013) Long-term follow-up of a spontaneously improving patient with junctional epidermolysis bullosa associated with ITGB4 c.3977-19T)A splicing mutation. Acta Derm Venereol 93:116-118

Dural O, Acar DK, Ekiz A, Aslan H, Polat İ, Yildirim G, Gulac B, Erdemoglu Y, Cay A, Hacihasanoglu O (2001) Prenatal ultrasound findings and a new ultrasonographic sign of epidermolysis bullosa with congenital pyloric atresia: a report of three cases. J Med Ultrason 41:495-498

Easterbrook PJ, Gopalan R, Berlin JA, Matthews DR (1991) Publication bias in clinical research. Lancet 337:867-872

Frew JW, Dopping-Hepenstal PJ, McGrath JA (2010) Categorizing immunoflourescence mapping in epidermolysis bullosa with pyloric atresia: use as a broad prognostic indicator. Australas J Dermatol 51:212-214 
Ha D, Idikio H, Krol A, Lin AN (1998) Junctional epidermolysis bullosa with pyloric atresia: a case with favourable outcome. J Cutan Med Surg 3:102-104

Hattori M, Shimizu A, Nakano H, Ishikawa O (2018) Mild phenotype of junctional epidermolysis bullosa with pyloric atresia due to a novel mutation of the ITGB4 gene. J Dermatol 45:e203-e204

Hicks TD, Singh H, Mikhael M, Shah AR (2018) Carmi syndrome in a preterm neonate: a multidisciplinary approach and ethical challenge. Case Rep Pediatr 2018:4548194

Hon KL, Li JJ, Cheng BL, Luk DC, Murrell DF, Choi PC, Leung AK (2015) Age and etiology of childhood epidermolysis bullosa mortality. J Dermatol Treat 26:178-182

Iacovacci S, Cicuzza S, Odorisio T, Silvestri E, Kayserili H, Zambruno G, Puddu P, D’Alessio M (2003) Novel and recurrent mutations in the integrin beta 4 subunit gene causing lethal junctional epidermolysis bullosa with pyloric atresia. Exp Dermatol 12:716-720

Inthorn J, Paul NW (2017) Das Kindeswohl als leitendes Prinzip. Pädiatrie 29:6-8

Joshi M, Krishnan L, Kuruvila S (2012) Large gastric perforation in carmi syndrome: a morbid complication in a rare association. J Neonatal Surg 1:57

Kayki G, Bozkaya D, Ozaltin F, Orhan D, Kaymaz F, Korkmaz E, Yigit S (2017) Epidermolysis bullosa with pyloric atresia and aplasia cutis in a newborn due to homozygous mutation in ITGB4. Fetal Pediatr Pathol 36:332-339

Kestenbaum B (2009) Case reports and case series. In: Kestenbaum B. Epidemiology and biostatistics. Springer, New York, S 22-25

Kidszun A, Linebarger J, Walter JK, Paul NW, Fruth A, Mildenberger E, Lantos JD (2016) What if the prenatal diagnosis of a lethal anomaly turns out to be wrong? Pedriatrics. https://doi.org/10.1542/ peds.2015-4514

Kim JH, Park HY, Lee HJ, Eom M, Choi EH (2011) Case of epidermolysis bullosa with pyloric atresia. Ann Dermatol 23(Suppl 1):S41-S44

Marjanovic Z, Slavkovic A, Djordjevic I (2013) Syndromic association of pyloric atresia and epidermolysis bullosa (Carmi syndrome) — a case report. West Indian Med J 62:149-151

Masunaga T, Niizeki H, Yasuda F, Yoshida K, Amagai M, Ishiko A (2015) Splicing abnormality of integrin $\beta 4$ gene (ITGB4) due to nucleotide substitutions far from splice site underlies pyloric atresiajunctional epidermolysis bullosa syndrome. J Dermatol Sci 78:61-66

Masunaga T, Ogawa J, Akiyama M, Nishikawa T, Shimizu H, Ishiko A (2017) Compound heterozygosity for novel splice site mutations of ITGA6 in lethal junctional epidermolysis bullosa with pyloric atresia. J Dermatol 44:160-166

Maurice P, Eyrolle-Guignot D, Dhombres F, Garel C, Gonzales M, Muller F, Jouannic JM (2013) The key role of ultrasound examination in the prenatal diagnosis of epidermolysis bullosa with pyloric atresia. Prenat Diagn 33:908-909

Mencía Á, García M, García E, Llames S, Charlesworth A, de Lucas R, Vicente A, Trujillo-Tiebas MJ, Coto P, Costa M, Vera Á, López-Pestaña A, Murillas R, Meneguzzi G, Jorcano JL, Conti CJ, Escámez Toledano MJ, del Río Nechaevsky M (2016) Identification of two rare and novel large deletions in ITGB4 gene causing epidermolysis bullosa with pyloric atresia. Exp Dermatol 25:269-274

Merrow AC, Frischer JS, Lucky AW (2013) Pyloric atresia with epidermolysis bullosa: fetal MRI diagnosis with postnatal correlation. Pediatr Radiol 43:1656-1661

Micheloni A, De Luca N, Tadini G, Zambruno G, D’Alessio M (2004) Intracellular degradation of beta4 integrin in lethal junctional epidermolysis bullosa with pyloric atresia. Br J Dermatol 151:796-802

Mutlu M, Kalay E, Dilber B, Aslan Y, Dilber E, Almaani N, McGrath JA (2015) Pyloric atresia-junctional epidermolysis bullosa syndrome showing novel c.4505-4508insACTC mutations in integrin b4 gene (ITGB4). Turk J Pediatr 57:385-387

Mylonas KS, Hayes M, Ko LN, Griggs CL, Kroshinsky D, Masiakos PT (2019) Clinical outcomes and molecular profile of patients with Carmi syndrome: a systematic review and evidence quality assessment. J Pediatr Surg 54:1351-1358

Nakano A, Pulkkinen L, Murrell D, Rico J, Lucky AW, Garzon M, Stevens CA, Robertson S, Pfendner E, Uitto J (2001) Epidermolysis bullosa with congenital pyloric atresia: novel mutations in the beta 4 integrin gene (ITGB4) and genotype/phenotype correlations. Pediatr Res 49:618-626

Natsuga K, Nishie W, Arita K, Shinkuma S, Nakamura H, Kubota S, Imakado S, Akiyama M, Shimizu H (2010) Complete paternal isodisomy of chromosome 17 in junctional epidermolysis bullosa with pyloric atresia. J Invest Dermatol 130:2671-2674

Nissen T, Wynn R (2014) The clinical case report: a review of its merits and limitations. BMC Res Notes $7: 264$

Oxford Centre for Evidence-based Medicine (2009) Levels of evidence. https://www.cebm.net/2009/06/ oxford-centre-evidence-based-medicine-levels-evidence-march-2009/. Zugegriffen: 24. Apr. 2020 
Ozge A, Safak H, Ebru H, Evrim U, Bilge SE, Leyla O, Kemal KA, Volkan B (2012) First successful preimplantation genetic diagnosis of epidermolysis bullosa with pyloric atresia: case study of a novel c.4505-4508insACTC mutation. J Assist Reprod Genet 29:347-352

Pfendner E, Uitto J (2005) Plectin gene mutations can cause epidermolysis bullosa with pyloric atresia. J Invest Dermatol 124:111-115

Pfendner EG, Lucky AW (2017) Epidermolysis bullosa with pyloric atresia. In: Adam MP, Ardinger HH, Pagon RA et al (Hrsg) GeneReviews ${ }^{\circledR}$ [Internet]. University of Washington, Seattle (https://www. ncbi.nlm.nih.gov/pubmed/20301336. Zugegriffen: 24. April 2020)

Pulkkinen L, Bruckner-Tuderman L, August C, Uitto J (1998a) Compound heterozygosity for missense (L156P) and nonsense (R554X) mutations in the beta4 integrin gene (ITGB4) underlies mild, nonlethal phenotype of epidermolysis bullosa with pyloric atresia. Am J Pathol 152:935-941

Pulkkinen L, Kim DU, Uitto J (1998b) Epidermolysis bullosa with pyloric atresia: novel mutations in the beta4 integrin gene (ITGB4). Am J Pathol 152:157-166

Pulkkinen L, Rouan F, Bruckner-Tuderman L, Wallerstein R, Garzon M, Brown T, Smith L, Carter W, Uitto J (1998c) Novel ITGB4 mutations in lethal and nonlethal variants of epidermolysis bullosa with pyloric atresia: missense versus nonsense. Am J Hum Genet 63:1376-1387

Ruzzi L, Gagnoux-Palacios L, Pinola M, Belli S, Meneguzzi G, D’Alessio M, Zambruno G (1997) A homozygous mutation in the integrin alpha6 gene in junctional epidermolysis bullosa with pyloric atresia. J Clin Invest 99:2826-2831

Salvestrini C, McGrath JA, Ozoemena L, Husain K, Buhamrah E, Sabery N, Leichtner A, Rufo PA, PerezAtayde A, Orteu CH, Torrente F, Heuschkel RB, Thomson MA, Murch SH (2008) Desquamative enteropathy and pyloric atresia without skin disease caused by a novel intracellular beta4 integrin mutation. J Pediatr Gastroenterol Nutr 47:585-591

Sarin YK, Nagdeve NG (2006) Carmi syndrome complicated by pharyngo-esophageal perforation. Indian Pediatr 43:61-64

Shevell MI (2004) The ethics of case reports. Paediatr Child Health 9:83-84

Singh S, Pant N, Rawat JD, Dheer Y (2017) Mucosal irregularity on gastric contrast study in a case of Carmi syndrome. J Neonatal Surg 6:54

Stoevesandt J, Borozdin W, Girschick G, Hamm H, Höcht B, Kohlhase J, Volz A, Wiewrodt B, Wirbelauer J (2012) Lethal junctional epidermolysis bullosa with pyloric atresia due to compound heterozygosity for two novel mutations in the integrin $\beta 4$ gene. Klin Padiatr 224:8-11

Takizawa Y, Shimizu H, Nishikawa T, Hatta N, Pulkkinen L, Uitto J (1997) Novel ITGB4 mutations in a patient with junctional epidermolysis bullosa-pyloric atresia syndrome and altered basement membrane zone immunofluorescence for the alpha6beta4 integrin. J Invest Dermatol 108:943-946

Trah J, Has C, Hausser I, Kutzner H, Reinshagen K, Königs I (2018) Integra ${ }^{\circledR}$-dermal regeneration template and split-thickness skin grafting: a therapy approach to correct aplasia cutis congenita and epidermolysis bullosa in carmi syndrome. Dermatol Ther (Heidelb) 8:313-321

Uitenbroek DG (2019) SISA Two by two table analysis. https:/www.quantitativeskills.com/sisa/statistics/ twoby2.htm. Zugegriffen: 24. April 2020

Wang JY, Marinkovich MP, Rieger KE (2019) Epidermolysis bullosa with pyloric atresia consistently demonstrates concurrent low intra-basal epidermal and lamina lucida cleavage planes: a survey of six cases. J Eur Acad Dermatol Venereol 34:e200-e203. https://doi.org/10.1111/jdv.16153 